BINARY NUCLEI OF PLANETARY NEBULAE

Agnès Acker

Observatoire de Strasbourg, France

In view of the spectral similarity and the probable evolutionary relation between certain planetary nebulae (PN) and other objects that are generally binaries (symbiotic stars, Be stars, or slow novae), it is probable that many nuclei of PN are double.

Astrometric observations (Cudworth, 1973) showed that six nuclei are binary. Also, 13 PN have a visible central star that is probably accompanied by a hotter star. If one counts other nuclei whose radial velocity and luminosity vary greatly, there are 36 nuclei in all that are probably double.

For seven of these nuclei, I took a series of spectra, in May and August, 1975, using two spectrographs with built-in image-tubes (77 and $90 \AA / \mathrm{mm}$, respectively). In three cases (Fg Sge, $\mathrm{BD}+66^{\circ} 1066$, and $\mathrm{BD}+30^{\circ} 623$, nuclei of $60-7^{\circ} 1,96+29^{\circ} 1$, and $165-15^{\circ} 1$, respectively), the radial velocity definitely varied, and for these a provisional period has been calculated.

ON THE VARIABILITY OF THE PLANETARY NEBULAE

\title{
E.B. Kostyakova
}

Sternberg State Astronomical Institute, U.S.S.R.

The study of several planetary nebulae, suspected earlier of variability, was continued during the last years at the Sternberg Astronomical Institute.

The photoelectric observations, carried out in 1968-1975 in Crimea and at the observatory Skalnate Pleso (Czechoslovakia), were examined together with the objective-prism spectrograms, obtained in a homogeneous way during several observing seasons in 1972-1975 with the 50-cm Maksutov telescope of the Crimean station.

The study confirmed the earlier conclusion ([1], and others) on the variability of planetaries NGC 6572 , 6891, IC 4997, and Hu2-1. The mentioned planetaries showed systematic changes of brightness in the range $0 m_{2}$ - $0 m 3$, both in photoelectric observations and in the spectral lines. 
The planetary nebula IC 3568, suspected earlier of variability, showed essentially no variations of brightness to either method of observations.

The nebula NGC 6721, measured as a whole, also appeared to be constant during the observed seasons of 1968-1975.

The detailed results will be published in Astr. Zh. U.S.S.R. References: 1. Kostyakova, E.B., Arhipova, V.P., and Savel'eva, M.V., Mém. Soc. Roy. Sci. de Liège, 6e sér. t.v, p. 473, 1973.

ULTRAVIOLET PHOTOMETRIC VARIATIONS IN THE CENTRAL STAR OF IC 418

D.P. Gilra, S.R. Pottasch, P.R. Wesselius, and R.J. Van Duinen University of Groningen, The Netherlands

Ultraviolet photometric observations of IC 418 taken with the Netherlands Astronomical Satellite (ANS) indicate variability in the central star brightness. The variations occurring on the time scales of 5 hours are only $4 \%$ at $3300 \AA$ but increase to about $15 \%$ at $1550 \AA$. The observations were not made frequently enough to determine a periodicity, if one exists. There are also $5-10 \%$ changes in brightness observed in A78 and NGC 6543, these are probably due to spectral changes in the central star spectra. About $10 \%$ type variations were found in VV 1-7 and He 2-131 but their interpretation is not clear.

A careful search failed to reveal any other variations $(>10 \%)$ in the planetary nuclei observed with ANS.

THE EFFECTIVE TEMPERATURE OF THE CENTRAL STAR, AND A CRITERION FOR COMPLETE ABSORPTION OF HYDROGEN IONIZING PHOTONS BY THE NEBULA

S.R. Pottasch, P.R. Wesselius, C.-C. Wu, and R.J. Van Duinen University of Groningen, The Netherlands

With the new measurements of the central star between $1500 \AA$ and $3300 \AA$, practically the entire continuous emission has been measured or can be deduced if the nebula is optically thick for hydrogen and helium ionizing radiation. The effective temperature can then be found directly, if the ratio of the radius to the distance of the star is known. This latter quantity can be determined with different assumptions: that the 